\title{
Application of Internet of Things Technology in Intelligent Transportation in Post-epidemic Era
}

\author{
Siqi He
}

\author{
Hohai University, Jiangsu Province Changzhou City, 213000, China \\ 1962410107@hhu.edu.cn
}

\begin{abstract}
As a new type of information technology, the Internet of Things is receiving extensive attention and research. In recent years, the Internet of Things technology has also been widely used in public transportation systems. The IoT is a good non-contact carrier during the epidemic period in 2021 , which plays a positive role in the prevention and control of COVID-19. Based on the previous IoT technology, intelligent transportation should be improved, innovated and applied in the post-epidemic period, which will be helpful to fight COVID-19 and protect people's safety.

This article will use the method of literature analysis to study the application of IoT technology in smart transportation, including 5G technology, vehicle tracking and detection technology, convolutional neural network, V2X communication technology, formation control technology and other technologies related to IoT. It makes a discussion that the above technologies can be applied to realize rational allocation of cases and medical resources, make full use of existing medical resources, and implement a coordinated and joint control system for major public health events.
\end{abstract}

Keywords: Internet of Things, Intelligent Transportation System, Formation Control, $5 G$ applications, Intelligent Intersection, Vehicle Tracking.

\section{INTRODUCTION}

In recent years, with the development of Internet of Things technology, transportation has become more and more intelligent, and the efficiency of transportation infrastructure has become greater. People, vehicles, and transportation infrastructure will all be closely connected. New impetus will be injected into the further development and improvement of intelligent transportation systems. The improvement of connectivity capabilities can allow the application of vehicle linkage to upgrade to unmanned driving, fleet scheduling and management, and traffic intelligence services. IoT technology can also make use of its ability of fast transmission and self-regulation to reduce the amount of data transmission, reduce the burden of computing, and increase the safety of intelligent transportation[1].

This paper mainly explains the connection between the IoT and smart transportation. During the epidemic prevention and control period, the existing IoT technology and the development and application of smart transportation will be used to explore the practical advantages and applications and problems of the
Internet of Things and future development prospects and development directions.

\section{THE DEFINITION AND CONNECTION OF IOT TECHNOLOGY AND INTELLIGENT TRANSPORTATION SYSTEM}

\subsection{Definition}

Internet of Things is the "Internet of all things connected", which combines a variety of information. It senses devices with the network to realize the interconnection of people, machines and things whenever and wherever possible. Its core is the interconnection of everything, especially between people and things and its basic features can be defined as overall perception, reliable transmission and intelligent processing. Information processing functions of the Internet of Things include acquiring, transmitting, processing and effecting information. It also provides high adaptability and increases intelligence to guarantee privacy and safety[2].

The intelligent transportation system can mainly 
improve the application requirements in four aspects: real-time traffic monitoring, public vehicle management, travel information service and vehicle auxiliary control. Data including vehicle location, speed, traffic flow, waiting time and the others is an important part of the form and development of the intelligent traffic. Through collecting a large amount of data real-time and accurate data analysis, it can record the user's daily activities and reflect the user's intention, preference and behavior patterns. And it can even make prediction of user's behavior.

\subsection{Connection}

The application of IoT technology in intelligent transportation includes video surveillance and acquisition technology, GPS technology, special short-range communication technology, location awareness technology, radio frequency identification and so on. It can change the transportation by changing the way of transportation systems collect and use data.

In the post-epidemic period, the application of Internet of Things technology in intelligent transportation also has a broad development direction.

\section{VARIOUS IOT TECHNOLOGIES IN INTELLIGENT TRANSPORTATION SYSTEMS}

\subsection{Intelligent traffic monitoring and tracking}

\subsubsection{Positioning Technology}

Table1 The comparation of five different positioning technology

\begin{tabular}{|c|c|c|c|c|c|}
\hline $\begin{array}{l}\text { Positioning } \\
\text { Technology }\end{array}$ & GPS & Wi-Fi & RFID & ZigBee & UWB \\
\hline principle & $\begin{array}{c}\text { Satellite } \\
\text { positioning }\end{array}$ & $\begin{array}{c}\text { Location } \\
\text { fingerprint } \\
\text { positioning }\end{array}$ & $\begin{array}{c}\text { Target } \\
\text { RFID tag } \\
\text { positioning }\end{array}$ & $\begin{array}{c}\text { Proximity } \\
\text { detection-and } \\
\text { multilateral } \\
\text { positioning }\end{array}$ & $\begin{array}{l}\text { Multilateral } \\
\text { positioning }\end{array}$ \\
\hline Service-Area & $\begin{array}{l}\text { Outdoor } \\
\text { open:area }\end{array}$ & $\begin{array}{l}\text { Wi-Fi. } \\
\text { coverage }\end{array}$ & $\begin{array}{c}\text { Limited.to- } \\
\text { acertain } \\
\text { place }\end{array}$ & $\begin{array}{c}\text { Organizational } \\
\text { network area } \\
\text { formed by } \\
\text { nodes }\end{array}$ & $\begin{array}{l}\text { Limited.to-" } \\
\text { UWB base- } \\
\text { station }\end{array}$ \\
\hline Advantage & $\begin{array}{l}\text { 1.high } \\
\text { precision } \\
\text { 2.high } \\
\text { coverage } \\
\text { 3.many } \\
\text { application } \\
\text { scenarios }\end{array}$ & $\begin{array}{l}\text { 1. fast } \\
\text { positioning } \\
\text { 2.high- } \\
\text { positioning } \\
\text { progress } \\
\text { 3.suitable for } \\
\text { indoor } \\
\text { positioning } \\
4 . \text { low'cost } \\
\text { 5.high- } \\
\text { coverage }\end{array}$ & \begin{tabular}{|l|} 
1.fast \\
recognition- \\
speed \\
2.strong \\
real-time \\
ability \\
3.easy to \\
control \\
4.high- \\
security
\end{tabular} & $\begin{array}{l}\text { 1.low power } \\
\text { consumption } \\
\text { 2.high- } \\
\text { capacity } \\
\text { 3.1owrate } \\
4.1 \text {. cost }\end{array}$ & $\begin{array}{l}\text { 1.high } \\
\text { intensive } \\
\text { reading } \\
2 . \text { low power } \\
\text { consumption } \\
\text { 3.high security }\end{array}$ \\
\hline Disadvantage & $\begin{array}{l}\text { 1.high cost } \\
\text { 2.many } \\
\text { blind spots } \\
3 \text {.low } \\
\text { reliability }\end{array}$ & $\begin{array}{l}\text { 1.affected by } \\
\text { the scope-of } \\
\text { services } \\
\text { 2.high power } \\
\text { consumption }\end{array}$ & $\begin{array}{l}\text { 1.small- } \\
\text { range } \\
\text { 2.big. } \\
\text { change. } \\
\text { with.label }\end{array}$ & $\begin{array}{l}\text { 1.10w datarate } \\
\text { 2.1ow } \\
\text { applicability }\end{array}$ & $\begin{array}{l}\text { 1.high cost } \\
\text { 2.susceptible- } \\
\text { to } \\
\text { environmental } \\
\text { influences }\end{array}$ \\
\hline
\end{tabular}

As is shown in table1, it is clear to find that GPS (outdoor) and Wi-Fi (indoor) have a much wider range of applications than the other three. However, it is hard to deny that they are more likely to be influenced by environment as well as UWB. People also should notice is that UWB and ZigBee can meet the need of long-time work because of the low consumption and RFID can be used to those important objects due to its processing power and operability. To conclude, making full use of those advantages to satisfy positioning requirements anywhere anytime is helpful for intelligent transportation.

\subsubsection{Tracking Technology}

Tracking technology can be divided into tracking of vehicles and tracking of persons.

Vehicle tracking and monitoring is using the video image processing methods to measure and detect vehicles in both time and space through parameters such as traffic flow, speed, lane change, and vehicle trajectory[3]. This type of technology can meet the requirements of detecting all types of vehicles, all types of traffic conditions and all lighting conditions, and real-time monitoring can also be achieved. Vehicle tracking systems can generate individual vehicle data to better model traffic flow and understand driver behavior.

Vehicle segmentation and tracking, and the computation of traffic parameters from the tracking data are the two most important stages in the tracking strategies[3].

User tracking and monitoring is to improve the safety of public traffic, which is to use SoftSIM to complete user tracking and conduct authenticated communication and identification[4]. It can provide all necessary functions provided in any hardware SIM and doesn't occupy any space on the device.

\subsection{Network communication technology}

\subsubsection{V2X technology}

$\mathrm{V} 2 \mathrm{~V}$ refers to vehicle-to-vehicle communication technology, and V2I refers to vehicle-to-infrastructure communication technology. Those two technologies can be used to solve various traffic problems.

Based on the MINMAX method, a street load balancing optimization model can be established, so as to maximize the use of vehicles and streets under different vehicle network conditions; Jain's fairness index and Jain's fairness index equation are used for balancing the congestion of each street in the Internet of Vehicles.

Among them, vehicle communication also needs to use GPS for vehicle positioning and vehicle location node acquisition, as well as roadside sensor intersection nodes and vehicle communication. $\mathrm{N}$ Parrado and $\mathrm{Y}$ Donoso used algorithms such as Global Congestion 
Algorithm, Modified Dijkstra Algorithm, Local-Congestion Based Algorithm and Natural Behavior Algorithm to establish vehicle and vehicle condition models, calculated Street Capacity, Origin/Destination Pairs for Each Vehicle and Approximate Flow, etc. And what mentioned above also compared and studied many factors such as efficiency, traveled distance and congestion to support the continuous change of the global state of the vehicle network and ensure the use of vehicle infrastructure in a more efficient manner[5].

\subsubsection{G technology}

In many developed countries, transportation accounts for $6 \%$ to $12 \%$ of the total population, which means that transportation infrastructure is under great pressure. And 5G technology can be applied to smart transportation and change smart transportation to alleviate traffic pressure.

The high reliability and high coverage of $5 \mathrm{G}$ technology can realize real-time information interaction and real-time monitoring by supporting sensor information exchange, which improves the automation level of public transportation and optimizes the economic benefits and sustainability of the transportation system[6].

\subsection{Vehicle and road condition management technology}

\subsubsection{Vehicle formation control}

The formation control of vehicles requires understanding of robot formation and communication methods, building a transmission system for robot coordinated movement based on the Internet of Things technology, and completing the collection of real-time external environmental information[7].

In the multi-robot formation control, using graph theory to understand the relationship between multiple robots and form a Laplacian matrix. It also can transform the transfer function into a state space model. Its aim is to get the controller canonical form and observer canonical form to obtain its controllable and observable gain, which are used to complete the formation control of vehicles. What should pay attention is that Leader-Follower applies linear consensus algorithm and uses the step response method to obtain the approximate value represented by the standard second-order model, which reasonably optimize the formation to improve the efficiency and accuracy of the mission[8].

Analyzing and building the operating environment of mobile robots, establishing a wireless communication network, and constructing a wireless sensor network are necessary steps to build The Internet of Things platform. Based on the IoT platform, deep learning will be used to solve the problem of collaborative navigation and control of multi-robots.

Robot systems also need to perceive the external environment in real time, including visual recognition, task management execution and assignment, behavior decision-making, etc. to achieve adaptation in various environments[7]. This kind of deep learning neural network algorithm based on particle swarm optimization can avoid obstacles to reach the destination quickly, accurately and efficiently.

\subsubsection{Vehicle and traffic prediction}

In order to realize real-time road traffic forecasting, much data needs to be collected for real-time forward-looking and accurate analysis. Meanwhile, people should pay attention to the number and types of integrity models and the computability of the models[9]. Because it is a real-time system, the data changes at every moment are great. In order to balance between parameter loss reduction and data accuracy improvement, people can use a multivariate spatial-temporal autoregressive (MSTAR) model to account for transient behavior on the traffic network and form continuous operation and uninterrupted real-time output generation.

\section{VARIOUS APPLICATIONS IN POST-EPIDEMIC ERA}

\subsection{Case tracking and monitoring}

\subsubsection{Real-time computer vision systems}

During the epidemic prevention period, hospitals and hotels will contact with suspected cases, infected cases or encounter isolation problems. In the process of using vehicles to transfer people, video tracking technology ensures remote monitoring of cases. And in terms of tracking the source of the epidemic, the trajectory of the case can be tracked in time, which can effectively prevent the spread of the epidemic.

\subsubsection{G technology}

Due to its high geographical coverage, privacy security and low delay, 5G technology can update all aspects of information of passengers in a timely manner. It also avoid the uncertainty of manual inspection and security risks caused by crowded people at public transportation exits.

5G technology has a strong ability of the network, supporting those who need to be synchronized and the application of real-time monitoring, automatic driving or automatic guided vehicle applications. Its low cost 
and short time synchronization to control remote cars can reduce the staff contact passengers on public transport during the period of the outbreak, and more safely and efficiently process the data.

\subsubsection{V2X technology}

V2X technology in intelligent transportation can use $5 \mathrm{G}$ information to interactively connect vehicles, roadside units and pedestrians, etc. During the epidemic period, it can use information interaction to share road conditions, locate all vehicle journeys of cases, and achieve the factual update and release of epidemic related information. Seeking help in time when meeting difficulties is another function, so that problems can be found, information can be transmitted and problems can be solved in time.

\subsection{Transportation and management of medical resources}

\subsubsection{Formation control}

Many countries in the world are faced with problems such as shortage of medical resources and medical personnel, and materials transportation difficulties due to inconvenience of public transportation. Formation control technology is an effective way to solve these problems. It can effectively transport abundant medical supplies to specific areas and alleviate problems of cross-regional medical treatment and patients in remote areas. And Leader-Follower technology can reduce the use and contact of transport personnel through remote control and in the transportation of materials on the basis of ensuring the safety of medical staff.

\subsubsection{Autonomous driving technology}

Regardless of whether it is during the epidemic or not, the transportation of materials is needed. Unmanned logistics vehicles represented by JD Logistics also played an important role in this epidemic. At present, JD.com's unmanned logistics delivery vehicle has been put into use in the Wuhan epidemic area, responsible for transporting medical supplies between hospitals, including masks and protective clothing, which can save human resources and reduce the risk of infection.

\subsection{Improvement of people's traffic safety}

\subsubsection{Prediction of traffic and vehicle}

Some bus stops and subway stations still have serious crowd congestion during rush hours, which wastes time and increases safety risks. During the epidemic, the multivariate spatial-temporal autoregressive (MSTAR) model is used to predict peak roads to achieve peak-shift travel. It contributes to ease traffic and crowd congestion and improve the safety of public transportation[9].

\subsubsection{Safety level certification for people using public transportation}

The embedded SIM card can be used for remote configuration, which can be applied to normal request and response commands.

It should be noticed that although people now have health codes and itinerary codes to judge the safety level of passengers, there are still some congestion at the exits and entrances of public transportation facilities such as train stations and bus stations due to the inconvenience of scanning codes. It may cause risks of spreading virus. Meanwhile, the manual inspection of entrances and exits will also lead to information inequity. What people must also consider is that many elderly and children cannot use these applications.

At this time, using SoftSIM technology can solve such problems well. In order to facilitate travel and ensure travel safety, all information can be transmitted to the SIM card or carry-on ID through real-time information interaction[4]. When taking public transportation, the ID information can be directly read to understand the user's itinerary, security situation, which reduces the risk of virus transmission, and provides convenient services for the elderly and children who cannot use the application.

\section{CONCLUSION}

Intelligent transportation plays a crucial role during epidemic period, and it is the center of the coordinated joint control system for major public health incidents. Through intelligent transportation applications, the rational allocation of cases and medical resources can be realized, and the existing medical resources can be fully utilized. The application of the coordinated joint control system for major public health incidents can make up for the inadequacy of the existing epidemic prevention and control system, optimize the prevention and control plan, and enhance the prevention and control capabilities. The combination of information technology, communication technology and resource optimization mathematical models are all helpful to realize the "early detection, early treatment, and early resolution" during the period. This greatly increase the response speed of sudden epidemics and improve accuracy and efficiency. Local epidemic spread controlling can be useful to control the scope of the epidemic. Through the application of smart transportation technology, seamless connection between patients and medical resources can not only gain time for rescue, but also greatly reduces the cost of rescue and impact of the epidemic on the economy and society. 


\section{ACKNOWLEDGMENTS}

Thanks to Professor Ding for his help in robot formation control, which enabled me to have a deeper understanding of formation control, leader follower and other aspects. I would also like to thank my thesis teacher, who has been providing me with a lot of help and advice in terms of writing methods, writing skills, writing content and so on. All of them help me to finish and improve my paper.

\section{REFERENCES}

[1] Luo, X. G. , Zhang, H. B. , Zhang, Z. L. , Yu, Y. , \& Li, K. . (2019). A new framework of intelligent public transportation system based on the internet of things. IEEE Access, PP(99), 1-1.

[2] Atzori, L., Iera, A., \& Morabito, G. (2010). The internet of things: a survey. Computer Networks, 54(15), 2787-2805.

[3] Coifman, B., Beymer, D., \& Mclauchlan, P. (1998). A real-time computer vision system for vehicle tracking and traffic surveillance. Transportation Research Part C, 6(4), 271-288.

[4] Yadav, S., \& Rishi, R. (2021). Secure and authenticate communication by using softsim for intelligent transportation system in smart cities. Journal of Physics: Conference Series, 1767(1), 012049 (10pp).

[5] Parrado, N., \& Donoso, Y. (2015). Congestion based mechanism for route discovery in a v2i-v2 $\mathrm{v}$ system applying smart devices and iot. Sensors, 15(4), 7768-806.

[6] Gohar, A., \& Nencioni, G. (2021). The role of 5g technologies in a smart city: the case for intelligent transportation system. Sustainability, 13.

[7] Guan, J. S. , Zhou, W. D. , Kan, S. B., , Sun, Y. , \& Liu, Z. B. . (2020). Robot formation control based on internet of things technology platform. IEEE Access, PP(99), 1-1.

[8] Ortega, G. , F Munoz, Quesada, E. , Garcia, L. R. , \& Ordaz, P. . (2015). Implementation of leader-follower linear consensus algorithm for coordination of multiple aircrafts. 2015 Workshop on Research, Education and Development of Unmanned Aerial Systems (RED-UAS). IEEE.

[9] Min, W., \& Wynter, L. (2011). Real-time road traffic prediction with spatio-temporal correlations. Transportation Research Part C: Emerging Technologies, 19(4), 606-616. 